\title{
THE USE OF CONVOLUTION NEURAL NETWORK ALGORITHM IN THE BIOLOGICAL IMAGE OF WEIGHTLIFTING OF SCAPULA DYSKINESIS
}

\author{
Sofia Julie \\ Faculty of Sport Sciences and Physical Education, Universidade de Coimbra, Spain. \\ *Corresponding Author Email:
}

This is an open access article distributed under the Creative Commons Attribution License, which permits unrestricted use, distribution, and reproduction in any medium, provided the original work is properly cited

\section{ARTICLE DETAILS}

\section{Article History:}

Received 16 November 2019 Accepted 20 Decmber 2019 Available online 8 January 2020

\begin{abstract}
In order to further realize the effect of scapula dyskinesis on weightlifters under the action of convolution neural network algorithm and biological image in weightlifting, in this study, the effect of scapula dyskinesis in weightlifting is studied by the way of weight lifting biological image and convolution neural network algorithm. The stability, strength and speed of the scapula under the snatch and the stability, strength and speed of the scapula under the clean and jerk are quantified to determine the effect of the dyskinesis of the scapula on the weightlifter under the action of the biological image and the convolution neural network algorithm. In this study, the biological images of each stage in the process of weightlifting are decomposed. Convolution neural network algorithm is used for data analysis, so as to realize the support and function of scapula for weight lifting. The results show that the weight lifting optimization effect based on the biological image and convolution neural network algorithm of scapula dyskinesis is the most significant. The stability, strength and speed of scapula of athletes have been improved obviously. It can be seen that the research method based on the biological image of weightlifting and convolution neural network algorithm has a good help for weightlifters. Conclusion: The effect of scapula dyskinesis under the influence of biological image and convolution neural network algorithm is studied. The stability, speed and strength of scapula of athletes in two different lifting ways are studied. The research shows that the method in this study has a very significant optimization and improvement for the weightlifting effect of athletes under different weightlifting methods. Through this study, it also shows that the effect improvement of weightlifting needs to be studied in many aspects, and a single level is unable to provide substantive help. This study greatly improves the realization of scapular dyskinesis.
\end{abstract}

\section{KEYWORDS}

Electromyography, scapula, weight lifting, strength, speed

\section{INTRODUCTION}

With the growing love for sports, weightlifting, football, basketball, badminton, swimming, volleyball and many other sports begin to enter people's lives. Especially with China's achievements in sports [1], people's enthusiasm for sports is very high. In all sports, weightlifting is a very good project in China. In particular, Chinese women's weightlifting performance is very outstanding. What impresses everyone most is the coherent steps in weightlifting, which is very aggressive and efficient. However, weight lifting is very demanding for the body [2]. Without correct guidance and practice, there is no way to achieve good results in actual combat [3]. In addition, weight lifting for the body consumption and loss is very large. If athletes can't recover and cultivate in time, it will be more and more difficult for athletes to do this action. Weightlifting has very strict requirements on speed, stability of scapula, and other aspects. Therefore, it is very important to keep healthy, optimize and improve the movement continuously [4]. For weightlifting, it is mainly snatch and clean and jerk. If the correct technology of the two aspects are not mastered, athletes will not be able to really improve their level in weightlifting, which is a relatively large learning problem encountered in current weightlifting activities [5]. If this problem cannot be solved effectively, the efficiency of weightlifting exercise is very low, and the progress is very slow. Therefore, this problem should arouse the attention and guidance of athletes and relevant departments, so as to find more efficient methods [6].
Therefore, based on this situation, in this study, it is proposed to optimize and improve the weight lifting effect based on scapular dyskinesis under the action of electromyography test, biological image and convolution neural network algorithm, so as to provide substantive help for the weight lifting exercise. The contribution of this study is mainly to use electromyography test to detect the obstacle of scapula, and then to provide valuable help for the improvement and recovery of weightlifters, and then to effectively solve the difficulties and obstacles caused by the way of weightlifting learning. The research of weightlifting is very important for the development of national fitness. In this way, people's physical quality can be improved correctly and effectively, and the performance of athletes in the competition can be improved [7].

To sum up, in this study, the effect of weight lifting of athletes is optimized and studied through the electromyography test, biological image and convolution neural network algorithm based on scapular dyskinesis. The research shows that under the effect of electromyography and convolution neural network algorithm, the improvement effect of scapula dyskinesis on the stability, speed and strength of scapula of athletes in two different lifting ways is more prominent, which improves the ability of athletes in lifting weights, and has an unusual significant optimization and improvement on lifting weights. The innovation of this study lies in the detailed classification and research of weightlifting, and the research on 
the relevant indicators and parameters of weightlifters, which is of great significance for the in-depth study of weightlifting. The results of this study still provide some guidance for future research, so this study is a valuable topic.

\section{METHODOLOGY}

\subsection{Research method}

In this study, the methods of biological graphics, literature and modeling are used to study and analyze the improvement effect of scapular dyskinesis analysis combined with electromyography test on weightlifting.

\subsection{Scapula and electromyography test}

Scapula plays an important role in the movement and function of shoulder joint. The movement of shoulder joint is always accompanied by the movement of scapula. The stability and coordination of scapula movement in all dimensions are of great significance to shoulder joint function and performance [8]. Electromyography test is a kind of noninvasive bioelectric signal which is recorded from the surface of human skin through electrodes when neuromuscular activity occurs. It has become one of the hot issues in competitive sports research and clinical rehabilitation.

\subsection{Convolutional neural network algorithm}

Convolution is a mathematical operation on two real valued functions. In practice, if the position of the moving entity at a certain time is represented by $x$, the smaller the time interval is, the higher the correlation of the measurement results is. Here, the weighted function w (a) is used to express. a is the time interval. In this way, the continuous estimation function s of the position of the moving entity can be obtained:

$s(t)=\int x(a) w(t-a) d a$

When time is integer value, the discrete form of convolution can be obtain ed:

$s(t)=\sum_{a=-\infty}^{\infty} x(a) w(t-a)$

In the field of image, convolutional neural network is used. Because of the two-dimensional nature of the picture, a two-dimensional core $\mathrm{K}$ is neede $\mathrm{d}$ :

$S(i, j)=(I, K)(i, j)=\sum_{m} \sum_{n} I(m, n) K(i-m, j-n)$

For the modeling of weightlifting action, a series of processing and analys is are needed for the input two-dimensional image of weightlifting, and th e coordinates of the actual weightlifting action corresponding to the featu re points in the image are finally obtained.

$\mu_{0}-\left(\frac{\omega}{2}\right) \mathrm{r} \leq \mu \leq \mu_{0}+\left(\frac{\omega}{2}\right) \mathrm{r}$

$\mathrm{v}_{0}-\left(\frac{\mathrm{h}}{2}\right) \mathrm{r} \leq \mathrm{v} \leq \mathrm{v}_{0}+\left(\frac{\mathrm{h}}{2}\right) \mathrm{r}$

In the equation, $r$ represents the ratio value retained in the weight lifting action depth map. The image depth of all weight lifting actions in some ar eas of this window is calculated as:

$g(x)=\frac{1}{1+e^{-x}}$

At this time, the image depth of each weight lifting action corresponds to a behavior. The average and variance of this variance set are calculated as follows:

$\tan (x)=\frac{e^{x}-e^{-x}}{e^{x}+e^{-x}}$
Loss $=F\left(x_{n}, W_{n}, b_{n}\right)$

According to the calculation results, the depth of the weight lifting action image corresponding to the variance is retained:

$\frac{\partial \text { Loss }}{\partial \mathrm{x}_{\mathrm{n}}}=\frac{\partial \mathrm{F}\left(\mathrm{x}_{\mathrm{n}}, \mathrm{W}_{\mathrm{n}}, \mathrm{b}_{\mathrm{n}}\right)}{\partial \mathrm{x}_{\mathrm{n}}}$

After the above-mentioned filtering processing, weight lifting action imag e is calibrated by camera. The calibration method based on 2D plane targ et is adopted. The calculation equation is as follows:

$H(x)=f(x, w)+W_{S} x$

The Euclidean distance is used to measure the similarity of weight lifting i mages. Its expression is

$\frac{\partial \mathrm{H}}{\partial \mathrm{x}}=\frac{\partial \mathrm{x}+\partial \mathrm{F}(\mathrm{x}, \mathrm{w})}{\partial \mathrm{x}}=1+\frac{\partial \mathrm{F}(\mathrm{x}, \mathrm{w})}{\partial \mathrm{x}}$

In the equation, i represents the feature vector number of the weight liftin $g$ action image. The smaller $D$ is, the closer the corresponding distance of $f$ eature points is, the higher the similarity is.

\subsection{Dynamic disturbance of scapula and its formation factors}

Scalar dyskinesis (SD) refers to the dysfunction of changing the position and trajectory of scapula in the process of resting position and dynamic movement. SD itself is not a shoulder injury, but a dysfunction related to the abnormal position of glenohumeral joint, acromioclavicular joint tension, space reduction under the acromion, muscle strength and activation sequence. The rehabilitation course of SD soft tissue elasticity weakening is shown in Figure 1.

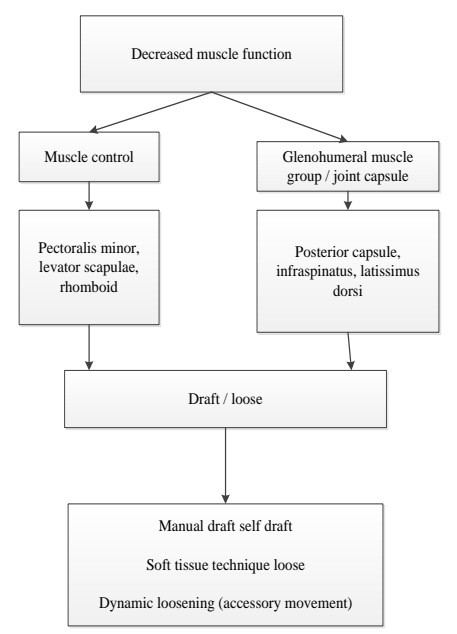

Figure 1: Rehabilitation course of SD soft tissue elasticity weakening

\subsection{Relationship between scapula and weightlifting}

Weightlifting belongs to the form of one-off maximum explosive force contraction. The driving force should form a whiplash movement with the core as the axis, and the small ring joint movement should be driven by the large link in the movement chain. With the help of the brake of the near side link, the momentum of the near side link can be transferred to the far side link, so as to increase the speed of the far side link and the speed of lifting weights. The stability of the scapula is improved, which can make the energy transfer on the power chain more smoothly and maximize the energy reaching the far side link. At the same time, the stability of the scapula provides stable support for the weight lifting of the upper limbs, which makes the muscles at the shoulder joint contract more fully, so as to improve the strength and speed of weightlifters.

\section{$2.6 \quad$ The improvement of weight lifting}

The method of analysis of scapular dyskinesis combined with electromyography is used to help athletes quickly and accurately find the types of scapular dyskinesis, so as to make a comprehensive rehabilitation 
and training plan, and help athletes improve their ability. The rehabilitation course of SD muscle function weakening is shown in Figure 2. Scapular stability training is to train the scapulae on both sides of the body at the same time, so that the scapulae on both sides of the body are in a relatively dynamic balance state, so as to provide more stable support for the movement of the upper limbs, so that the energy transfer on the power chain is more smooth, so as to maximize the energy reaching the far side, so as to significantly improve the result of throwing the solid ball forward on the head of both hands.

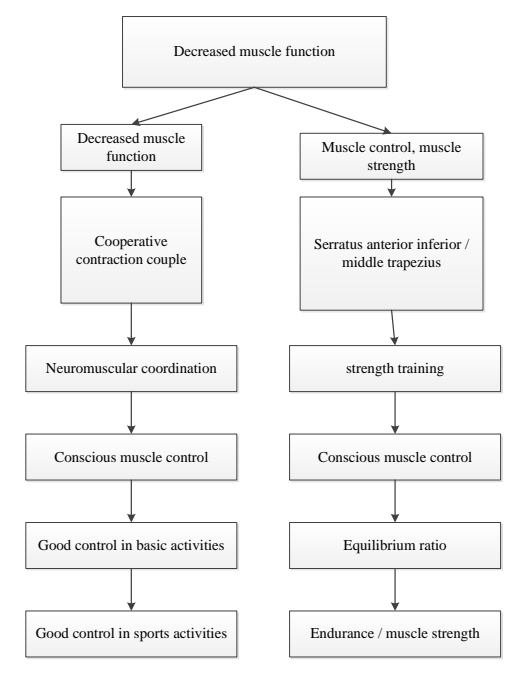

Figure 2: Rehabilitation course of SD muscle function weakening

After the experiment and before the experiment, the stability of the scapulae reflects the speed and strength of weightlifters, and there is a significant difference between the test results. The results show that after 8 weeks of scapulae stability training, the experimental group's scapulae stability, strength and speed of weightlifters are improved. It shows that scapulae stability training can effectively improve the speed and strength of weightlifters. The results show that the analysis of scapular dyskinesis combined with electromyography test can significantly improve the stability, speed and strength of the scapula of the athletes under two different weightlifting modes, improve the ability of the athletes in weightlifting, and significantly optimize and improve the weightlifting effect of the athletes.

\section{RESULTS AND DISCUSSION}

The stability analysis of scapula in snatch state is shown in Figure 3, and the biological image in snatch state is shown in Figure 4. From the data in the figure, it can be clearly seen that the scapulae stability of weightlifters based on the scapular dyskinesis under the effect of electromyography test and convolution neural network algorithm has been significantly improved compared with that before the experiment. In the weightlifting competition, the strength requirement of shoulder is very high, and the stability of scapula plays a very important role in weightlifting. It can be seen that the electromyography test and convolution neural network algorithm based on scapular dyskinesis have a significant effect on the optimization and help of weightlifting. From the biological image in the snatch state in Figure 4, it can be seen that the whole process is divided into five steps: preparation, wrists rolling, standing, snatching and completion. The correct use of these steps can grasp the key link of snatch.

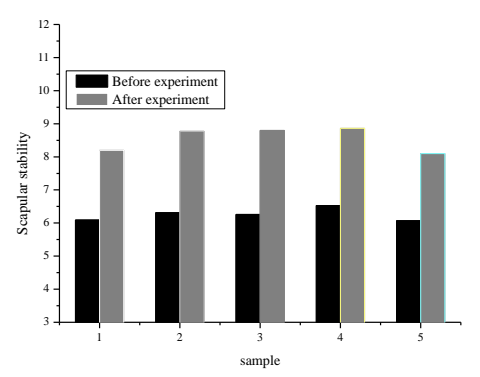

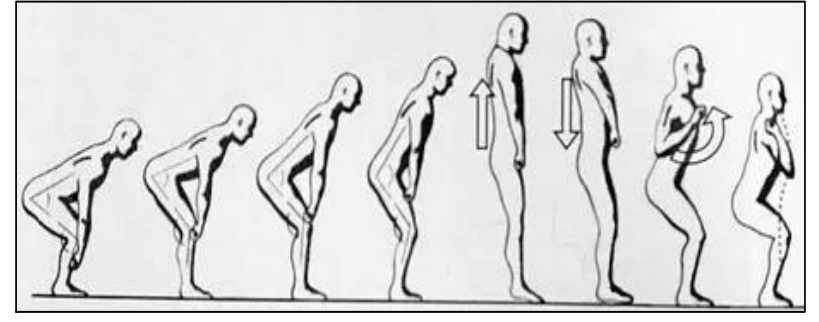

Figure 4: Biological images in snatch state

The analysis of lifting speed in snatch state is shown in Figure 5, and the biological image in snatch state is shown in Figure 6. From the data in the figure, it can be seen that the speed of weightlifting of athletes has been significantly improved in the five groups of experiments in this study based on scapular dyskinesis under the effect of electromyography test and convolution neural network algorithm. In weightlifting, there are very strict requirements for speed, because speed can guarantee the explosive force of the whole movement. Therefore, it can be seen that the speed effect in weightlifting is improved and the ability of athletes is improved based on the electromyography test and convolution neural network algorithm of scapular dyskinesis. From the biological image in the snatch state in Figure 6, it can be seen that the whole process is roughly divided into three steps: standing, snatching and completion. The proficient study of these steps can effectively improve the ability of weightlifting

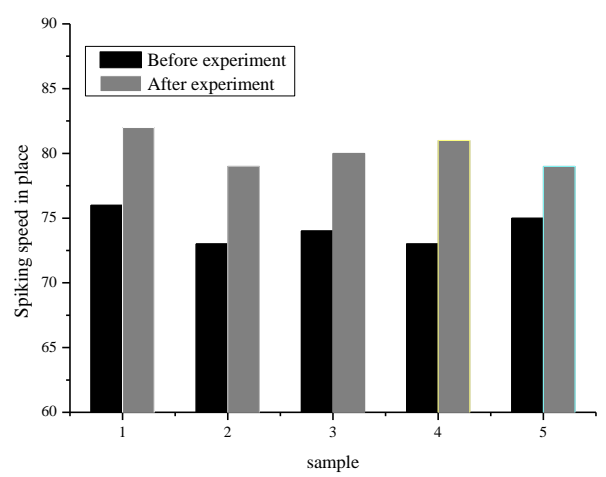

Figure 5: Analysis of weight lifting speed in snatch state

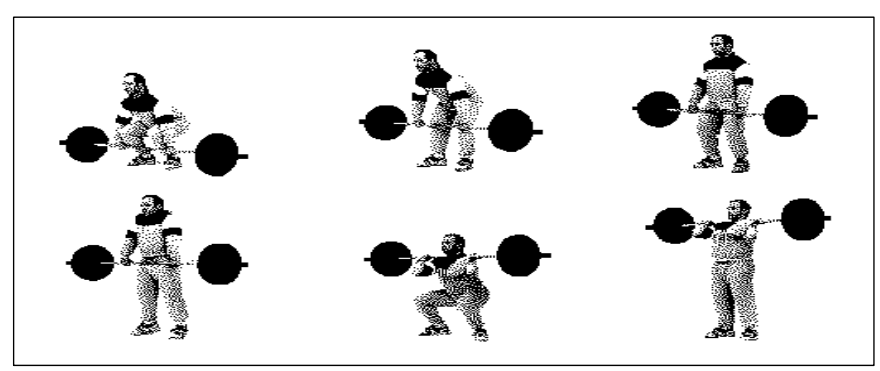

Figure 6: Biological image in snatch state

The barbell displacement analysis in clean and jerk state and the biological image in clean and jerk state are shown in Figure 7 and Figure 8. It can be clearly seen from the figure that the displacement of weightlifters has been significantly improved in the five groups of experiments conducted in this study based on scapular dyskinesis under the effect of electromyography test and convolution neural network algorithm. In weightlifting, there is a high demand for strength. Displacement is a very important parameter in the study of force. The displacement has a very important influence on the lifting effect. It can be seen that based on scapular dyskinesis under the effect of electromyography test and convolution neural network algorithm, the strength of weightlifters can be improved and the weightlifting displacement of athletes can be improved. From the biological image of volleyball single jump spike in Figure 8, it can be seen that the whole process is divided into five steps: start, squat and finish. Through these steps, the lifting capacity of clean and jerk can be significantly improved.

Figure 3: Stability analysis of scapula in snatch state 


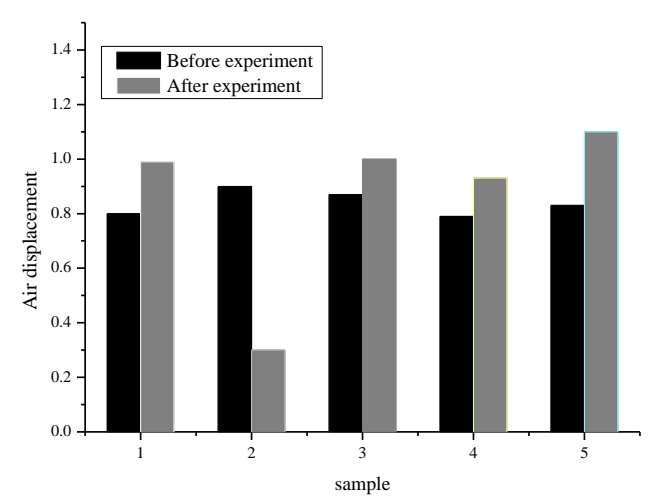

Figure 7: Analysis of displacement in clean and jerk state

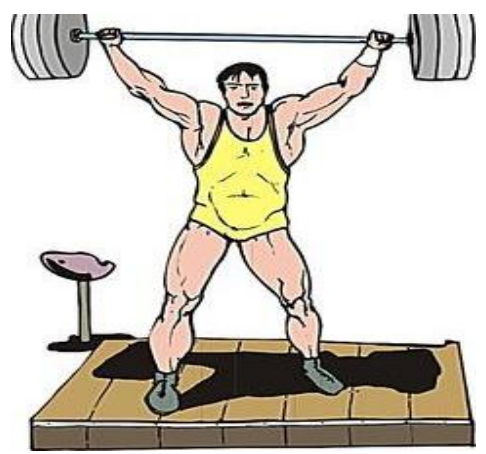

Figure 8: Biological image in clean and jerk state

\section{CONCLUSION}

In this study, the optimization and improvement effects of two different lifting methods are studied by using electromyography, biological image and convolution neural network algorithm based on scapular dyskinesis. Biological images clearly reflect the movement and shape of athletes. The results show that the optimization effect of scapular dyskinesis on weight lifting is significant under electromyography test, biological image and convolution neural network algorithm. No matter for snatch or clean and jerk, the stability, strength and speed of scapulae of athletes have been improved obviously. In addition, the data of scapular dyskinesis in this study obtained by electromyography test, biological image and convolution neural network algorithm are authentic. The optimization effect of scapular dyskinesis on weight lifting is significant under electromyography test and biological image.
Therefore, in this study, weight lifting is improved by electromyography test, biological image and convolution neural network algorithm based on scapular dyskinesis. The results show that the method of this study is multi-angle and multi-level for the improvement of athletes in weightlifting, and has achieved a comprehensive improvement effect. This study is also limited by some limitations, such as: the analysis of weightlifting is a more complex work, involving multiple levels of cooperation. In the algorithm analysis, some factors are idealized to reduce the interference. Therefore, the results are a little less convincing. Follow up research can be more in-depth and detailed, more comprehensive, which will reduce the interference of some other factors. This study has important reference value for later researchers.

\section{REFERENCES}

[1] Jiang, G., He, H., Yan, J. 2018. Multiscale convolutional neural networks for fault diagnosis of wind turbine gearbox. IEEE Transactions on Industrial Electronics, 66 (4), pp. 3196-3207.

[2] Peeters, C., Guillaume, P., Helsen J. 2018. Vibration-based bearing fault detection for operations and maintenance cost reduction in wind energy. Renewable Energy, 116, pp. 74-87.

[3] Ziaja, A., Antoniadou, I., Barszcz, T. 2016. Fault detection in rolling element bearings using wavelet-based variance analysis and novelty detection. Journal of Vibration and Control, 22(2), pp. 396-411.

[4] Feng, Z., Qin, S., Liang, M. 2016. Time-frequency analysis based on Vold-Kalman filter and higher order energy separation for fault diagnosis of wind turbine planetary gearbox under nonstationary conditions. Renewable Energy, 85, pp. 45-56.

[5] Chen, J., Pan, J., Li, Z. 2016. Generator bearing fault diagnosis for wind turbine via empirical wavelet transform using measured vibration signals. Renewable Energy, 89, pp. 80-92.

[6] Wang, T., Han, Q., Chu, F. 2019. Vibration based condition monitoring and fault diagnosis of wind turbine planetary gearbox: A review. Mechanical Systems and Signal Processing, 126, pp. 662-685.

[7] Maheswari, R.U., Umamaheswari, R. 2017. Trends in non-stationary signal processing techniques applied to vibration analysis of wind turbine drive train-A contemporary survey. Mechanical Systems and Signal Processing, 85, pp. 296-311.

[8] Astolfi, D., Scappaticci, L., Terzi, L. 2017. Fault diagnosis of wind turbine gearboxes through temperature and vibration data. International Journal of Renewable Energy Research (IJRER), 7(2), pp. 965-976 